\title{
Identificación de procesos patológicos más frecuentes en fachadas y cubiertas en España a partir del método Delphi
}

\section{Most common pathological processes identification in facades and roofs in Spain from Delphi method}

L. Ortega $^{(*)}$, B. Serrano-Lanzarote ${ }^{(* *)}$, J. M. ${ }^{\text {a }}$ Fran-Bretones ${ }^{(* *)}$

\begin{abstract}
RESUMEN
Los métodos basados en factores de durabilidad, según ISO-15686, son una forma eficiente de estimar la vida útil los elementos de un edificio. Sin embargo, se ha constatado la escasa información sobre cuantificación de dichos factores y sobre su vinculación con la vulnerabilidad o procesos de deterioro que podría presentar un elemento constructivo. En consecuencia, este artículo describe un procedimiento para caracterizar los mecanismos de daño más frecuentes en la envolvente del edificio, por ser un elemento con un elevado riesgo de presentar lesiones y ver reducida su durabilidad. Para elaborar dicha información se ha utilizado el método Delphi que implica la consulta a grupos de expertos a fin de generar convergencia de opiniones. Los resultados obtenidos permiten la identificación y cuantificación de los factores de durabilidad relevantes que condicionan la aparición de un daño de un elemento y afectan a su vida útil.
\end{abstract}

Palabras clave: Lesiones constructivas; patología constructiva; durabilidad; vulnerabilidad; vida útil; vida de servicio; método Delphi.

\section{ABSTRACT}

The estimated service life, as well as its components materials, is critical in order to establish realistic building maintenance programs, manageable and economically viable. The methods based on durability factors, proposed in ISO15686, are an efficient way of estimating the building's service life. However, there has been limited information on quantifying the durability values, and their relationship to vulnerability or processes of deterioration which might present a constructive element. Then, this paper describes a method to characterize the most frequent damage mechanisms in the envelope of a building, since it is an element with a high risk of lesions and, accordingly, with a reduced durability. The system used to generate this information was the Delphi method that involves consultation to groups with expert-based approach to generate convergence of views. The results allow the identification and quantification of relevant durability factors that determine the occurrence of damage to an element affecting its service life.

Keyword: Building lesions; construction pathology; durability; vulnerability; service life; Delphi method.

(*) Instituto Valenciano de la Edificación, Valencia (España).

(**) Universitat Politècnica de València, (España).

Persona de contacto/Corresponding author: lortega@five.es (L. Ortega)

Cómo citar este artículo/Citation: Ortega, L., Serrano-Lanzarote, B., Fran-Bretones, J. M. ${ }^{\text {a }}$ (2015). Identificación de procesos patológicos más frecuentes en fachadas y cubiertas en España a partir del método Delphi. Informes de la Construcción, 67(537): eo67, doi: http://dx.doi.org/10.3989/ic.13.144.

Licencia / License: Salvo indicación contraria, todos los contenidos de la edición electrónica de Informes de la Construcción se distribuyen bajo una licencia de uso y distribución Creative Commons Reconocimiento no Comercial 3.o. España (cc-by-nc). 


\section{INTRODUCCIÓN}

Tal y como afirman Moser y Hovde en su estudio Performance based methods for service life prediction (1), en los últimos años se ha percibido un aumento en el interés por determinar la durabilidad y la vida útil de los elementos constructivos de los edificios, así como de sus materiales y componentes. Este interés está justificado tanto por cuestiones económicas como medioambientales. Entre las cuestiones económicas cabe destacar el gran número de edificios existentes, mayoritariamente construidos en la segunda mitad del siglo XX, que entendidos como capital inmobiliario, representan una cantidad importante del capital nacional de los países. También es necesario recordar que los costes anuales de inspección y mantenimiento de un edificio representan un peso importante en la economía de un país o en la competitividad de una industria o empresa. Respecto a las cuestiones medioambientales no hay que olvidar la escasez de recursos materiales y energéticos, así como el impacto medioambiental causado por los edificios.

La durabilidad está directamente relacionada con la búsqueda de un desarrollo sostenible. En Europa, se ha demostrado que el sector de la construcción ha sido el responsable aproximadamente de:

- El $40 \%$ del material de consumo.

- $\mathrm{El}$ 40\% del consumo de energía.

- El 40\% de los depósitos de residuos en los vertederos.

- El 35\% de las emisiones de gases efecto invernadero. ${ }^{1}$

A la vista de estos datos, incluso una reducción limitada en el consumo de materiales o de energía, o en la generación de residuos, representaría ahorros totales significativos que podrían tener un gran impacto en el conjunto de la sociedad. Se espera que una importante contribución a los cambios en este ámbito se deba a la concienciación de la influencia de la durabilidad de los materiales y sistemas y de cuán importante es el establecer métodos fiables para la cuantificación de la durabilidad y para la predicción de la vida útil. Cada vez más, los fabricantes de materiales y sistemas constructivos son conscientes de esta problemática y buscan métodos para evaluar el riesgo prematuro de deterioro de sus productos debido a unas condiciones específicas.

Cómo no podemos conocer la vida útil con antelación, el reto pasa a ser el conseguir una predicción de la vida útil lo más fiable posible, utilizando los datos de los que se disponen actualmente.

Monjo en el artículo «Durabilidad vs Vulnerabilidad»(2) establece que la durabilidad de un elemento constructivo es función de su vulnerabilidad, entendida como el conjunto de debilidades o posibles procesos patológicos, que presenta un elemento constructivo al quedar expuesto a las acciones exteriores previsibles durante su vida útil. Tomando como punto de partida el enfoque que introduce Monjo en su artículo, el primer paso para establecer los factores que afectan a la durabilidad de fachadas y cubiertas, es estudiar su vulnerabilidad, es decir, aquellos procesos de deterioro a los que más pueden verse expuestos. Por ello, el trabajo de investigación que a continuación se expone pretende identificar las lesiones que más comúnmente afectan a los elementos constructivos de la envolvente de los edificios, así como aquellos factores que puedan favorecer o evitar la aparición de cada una de las lesiones estudiadas.

Para ello, en primer lugar, se ha llevado a cabo un estudio de la bibliografía existente en relación a los procesos patológicos más frecuentes en fachadas y cubiertas, cuyos resultados se exponen a continuación, en el apartado 2.1. Finalmente ha sido necesario recurrir al empleo del método Delphi para completar la información obtenida por las razones que se justifican en el apartado 5.2 del presente artículo.

\section{ANTECEDENTES}

\subsection{Estado de la cuestión}

Recurriendo al enfoque que introduce Monjo en su artículo (2), el primer paso de cara a establecer los factores que afectan a la durabilidad de fachadas y cubiertas, es determinar su vulnerabilidad, es decir, aquellos procesos de deterioro que más probablemente les puedan afectar. Para ello, en primer lugar, se ha llevado a cabo un análisis de la bibliografía y estudios existentes en relación a los procesos patológicos más frecuentes en fachadas y cubiertas, cuyos resultados se exponen a continuación.

\subsubsection{Patología de fachadas urbanas}

El primer estudio (3) que se ha considerado relevante en relación a la materia fue llevado a cabo por diversos profesores del Departamento de Construcción de la Escuela Técnica Superior de Arquitectura de la Universidad de Valladolid en colaboración con los alumnos de $4^{\circ}$ año del curso 1982/83. En el estudio se analizaron 1915 casos de lesiones constructivas de fachadas ubicadas en la ciudad de Valladolid, con el siguiente resultado estadístico, reflejado en la Tabla 1.

El detalle de estos resultados, y su análisis, está recogido en la publicación que fue consecuencia de este trabajo titulada: Patología de fachadas urbanas (3).

\subsubsection{Estudio de la Cátedra de Construcción IV de la Escuela Técnica Superior de Arquitectura de la Universidad Politécnica de Madrid}

Se exponen a continuación los resultados de un trabajo llevado a cabo en la Cátedra de Construcción IV de la Escuela Técnica Superior de Arquitectura de la Universidad Politécnica

Tabla 1. Porcentaje de lesiones presentes en las fachadas estudiadas (3).

\begin{tabular}{|l|c|}
\hline \multicolumn{1}{|c|}{ Lesión } & Porcentaje \\
\hline Desprendimientos & $43 \%$ \\
\hline Suciedades & $25 \%$ \\
\hline Grietas y fisuras & $15 \%$ \\
\hline Humedades & $7 \%$ \\
\hline Oxidaciones & $6 \%$ \\
\hline Erosiones & $4 \%$ \\
\hline
\end{tabular}

\footnotetext{
${ }^{1}$ Los porcentajes relativos al sector de la construcción en Europa son datos aportados por la Plataforma Tecnológica Española de Construcción (www.plataformaptec.com) en el documento Visión estratégica 2025 (Plataforma Tecnológica Española de la Construcción 2010)
} 
de Madrid con la colaboración del profesor Rodolfo Hernando Cotarelo y la participación de alumnos de $6^{\circ}$ curso, en los años 1988,89 y 90 (4). En el estudio se recogieron un total de 5000 fichas de lesiones de fachadas de la ciudad de Madrid, que se analizaron informáticamente mediante un programa de ordenador diseñado al efecto. Los resultados que se obtuvieron se presentan a continuación en la Tabla 2.

Tabla 2. Porcentaje de lesiones presentes en las fachadas estudiadas en la ciudad de Madrid (4).

\begin{tabular}{|l|c|}
\hline \multicolumn{1}{|c|}{ Lesión } & Porcentaje \\
\hline Desprendimientos & $23 \%$ \\
\hline Suciedades & $22 \%$ \\
\hline Grietas y fisuras & $15 \%$ \\
\hline Humedades & $13 \%$ \\
\hline Erosiones & $9 \%$ \\
\hline Corrosiones & $8 \%$ \\
\hline Eflorescencias & $5 \%$ \\
\hline Organismos & $5 \%$ \\
\hline
\end{tabular}

Los datos de este estudio están contenidos en un banco de datos patológicos, en el Departamento de Construcción y Tecnología Arquitectónicas de la Universidad Politécnica de Madrid.

\subsubsection{Siniestralidad arquitectónica}

Joseba Escribano Villán publicó en 1994 un resumen de su tesis doctoral sobre siniestros en edificación denominado $\mathrm{Si}$ niestralidad arquitectónica (5). El estudio está basado en los datos de siniestros producidos en la edificación desde 1984 a 1987 , sobre un total de 1820 expedientes pertenecientes a la recién creada Mutua de Seguros de Arquitectos Superiores ASEMAS. El enfoque está orientado a conocer las responsabilidades de la siniestrabilidad. Este trabajo concluye con unas propuestas de ámbito político y legal que, vistas ahora en perspectiva, resultan cuanto menos muy predictivas, como es la necesidad de una Ley de Edificación Española, la reforma del Código Civil y la limitación del ámbito de las responsabilidades, la creación de un Seguro Obligatorio para todos los agentes de la construcción, la necesidad de un Libro del Edificio con sus manuales de mantenimiento y la necesidad de desarrollar las Normas Tecnológicas del momento, endureciéndolas y convirtiéndolas en obligato$\operatorname{rias}(6)$.

En el último capítulo del libro se incluye un extracto que recoge las causas de los siniestros que se producen con mayor frecuencia y que a la vez, suponen un mayor costo social y económico. La proporcionalidad que expone el autor de los principales síntomas denunciados queda recogida en la Tabla 3.

\subsubsection{Campaña «La casa en forma»}

En el año 2000, el Colegio Oficial de Aparejadores y Arquitectos Técnicos de Murcia llevó a cabo, en el municipio de Murcia, una campaña con el lema «La casa en forma». La campa tenía dos objetivos principales:

- Sensibilizar sobre la necesidad de mantenimiento, ofreciendo información a los usuarios.

- Defender los intereses del usuario, realizando inspecciones técnicas de edificios de más de veinte años.
Tabla 3. Datos extraídos de la gráfica sobre la sintomatología general detectada en los sinestros contenida en la publicación Siniestralidad arquitectónica (5).

\begin{tabular}{|l|c|}
\hline \multicolumn{1}{|c|}{ Lesión } & Porcentaje \\
\hline Humedades & $37,5 \%$ \\
\hline Fisuración & $25,5 \%$ \\
\hline Acabados & $9 \%$ \\
\hline Estructura & $6 \%$ \\
\hline Otros & $\mathbf{2 2 \%}$ \\
\hline
\end{tabular}

Durante la campaña se enviaron arquitectos técnicos para realizar, de forma gratuita, 80 inspecciones técnicas de los elementos exteriores del edificio.

Como datos más significativos de dicho estudio cabe señalar que la lesión que más se produce es la de roturas y degradación de los elementos (en el $92 \%$ de los edificios en fachadas y en el $75 \%$ en cubiertas). En el caso de fachadas destacan también las humedades y las fisuras y grietas, (65\% y $60 \%$ respectivamente). Sin embargo en cubiertas predominan esas mismas dos lesiones, pero en diferente proporción (35\% y $62 \%$, respectivamente). En cuanto a las humedades, es interesante observar que los autores apuntan que, en muchos casos, aparecen a causa de la degradación de otros elementos tales como jardineras y vierteaguas en fachadas y juntas de dilatación, así como sumideros y albardillas en cubiertas (7).

El estudio también concluía que las roturas son el principal problema de los aplacados en fachadas, mientras que en fábrica vista y en revestimientos continuos las humedades aparecen en mayor número.

\subsubsection{Rehabilitación y mantenimiento de edificios}

Pascual Úbeda presentó en el libro Rehabilitación y mantenimiento de edificios (8) un estudio estadístico sobre patología en los edificios con datos obtenidos de una institución dedicada al seguro de profesionales. La muestra que se usó en el estudio consistió en 2717 casos de reclamaciones, distribuyéndose, según la información recogida en la Tabla 4.

\subsubsection{Premios de calidad en la edificación de la Región de Murcia}

En la región de Murcia desde el año 2004 y cada dos años se celebran los Premios de Calidad en la Edificación de la Región de Murcia. Estos premios ponen en valor la calidad de los edificios premiando, tanto al edificio en su conjunto por el compromiso de sus propietarios con su conservación, como la durabilidad y criterios de selección de las soluciones constructivas y de los materiales empleados, reconociendo de esta forma la labor tanto de los profesionales como de los usuarios.

De las cuatro ediciones celebradas hasta el momento, en las tres últimas se ha publicado un anexo técnico vinculado a cada convocatoria, cuyo objetivo responde al estudio de las soluciones constructivas adoptadas en edificios cuya antigüedad era superior a 15 años que hubieran soportado el paso del tiempo sin alterar sus prestaciones y con costos de mantenimiento razonables. Este estudio se realiza sobre una muestra de edificios correspondientes a los candidatos de todas las ediciones anteriores. Con este análisis los organizado- 
Tabla 4. Número de reclamaciones presentadas sobre cada lesión (8).

\begin{tabular}{|l|c|c|}
\hline Lesión & No de reclamaciones & Porcentaje \\
\hline Otras causas patológicas & 789 & $29,03 \%$ \\
\hline Humedades. Fallo impermeabilización cubierta & 65 & $24,21 \%$ \\
\hline Fallos estructurales & 384 & $14,13 \%$ \\
\hline Desprendimiento del revestimiento de fachada & 159 & $5,85 \%$ \\
\hline Daños por flexibilidad estructural & 134 & $4,93 \%$ \\
\hline Fallo de otras instalaciones & 113 & $4,15 \%$ \\
\hline Excavación excesiva & 85 & $3,12 \%$ \\
\hline Fallo del suelo (otras causas) & 72 & $2,64 \%$ \\
\hline Fallo de la instalación de fontanería & 60 & $2,20 \%$ \\
\hline Fallo del suelo (rellenos) & 58 & $\mathbf{2 , 1 3} \%$ \\
\hline Fallo en la red de saneamiento & $47,72 \%$ \\
\hline Humedades. Fallo de bajantes y desagües & 46 & $1,69 \%$ \\
\hline Fallo del suelo (arcillas expansivas) & 39 & $1,43 \%$ \\
\hline Humedades que provienen del suelo & 39 & $1,43 \%$ \\
\hline Desprendimiento de tierras & 23 & $0,84 \%$ \\
\hline Fallo de la instalación del ascensor & 6 & $0,22 \%$ \\
\hline Fallo de la instalación eléctrica & 5 & $0,18 \%$ \\
\hline TOTAL & $\mathbf{2 7 1 7}$ & $\mathbf{1 0 0 \%}$ \\
\hline
\end{tabular}

res pretenden resaltar las soluciones constructivas que mejor han soportado el paso del tiempo y señalar, por otra parte, los daños más frecuentes. En el anexo técnico correspondiente a la última edición se incluye la Tabla 5 .

De la tabla podemos extraer que la lesiones que más afectan a las fachadas analizadas son la degradación del material, las manchas y los grafitis, y las que más afectan a las cubiertas analizadas, son la degradación de material, la presencia de organismos y la suciedad.

\subsubsection{Estadísticas de siniestralidad de la edificación en la Región de Murcia}

La publicación Manual prevención de fallos: Estanqueidad en fachadas (10) expone las conclusiones de un estudio de las estadísticas de siniestralidad de la edificación en la Región

Tabla 5. Daños en función del elemento constructivo (9).

\begin{tabular}{|c|l|c|}
\hline \multicolumn{3}{|c|}{ Fachada (paño ciego) } \\
\hline Orden & \multicolumn{1}{|c|}{ Lesión } & Porcentaje \\
\hline 1 & Degradación del material & $29 \%$ \\
\hline 2 & Manchas & $27 \%$ \\
\hline 3 & Desprendimientos & $6 \%$ \\
\hline 4 & Fisuras & $4 \%$ \\
\hline 5 & Grafitis & $22 \%$ \\
\hline 6 & Grietas & $4 \%$ \\
\hline 7 & Organismos & $2 \%$ \\
\hline 8 & Oxidación & $6 \%$ \\
\hline \multicolumn{2}{|c|}{ Lesión } \\
\hline Orden & \multicolumn{2}{|c|}{} \\
\hline 1 & Degradación del material & $30 \%$ \\
\hline 2 & Manchas & $7 \%$ \\
\hline 3 & Desprendimientos & $3 \%$ \\
\hline 4 & Fisuras & $3 \%$ \\
\hline 5 & Grietas & $3 \%$ \\
\hline 6 & Organismos & $27 \%$ \\
\hline 7 & Suciedad & $7 \%$ \\
\hline 8 & Humedades & \\
\hline
\end{tabular}

de Murcia, que se llevó a cabo en colaboración con el Colegio Oficial de Aparejadores, Arquitectos Técnicos e Ingenieros de Edificación de la Región de Murcia. En la publicación se muestra la distribución de los siniestros en función del tipo de daño tal como se muestra en la Figura 1.

De la gráfica se puede deducir que las humedades y las grietas y fisuras son los daños más comunes.

\subsubsection{Análisis de los datos obtenidos}

Atendiendo a los estudios analizados y en términos generales, las conclusiones más relevantes son:

1) Las lesiones que más afectan a las fachadas, son las humedades, las fisuras y grietas y los desprendimientos.

2) Las lesiones que más afectan a las cubiertas, son las humedades y las fisuras y grietas.

3) El hecho de que los estudios que se han analizado sean, bien estudios locales, o bien se basen en datos procedentes de compañías de seguros, hace que los resultados obtenidos por un lado, en el caso de los estudios locales, no



Figura 1. Distribución de siniestros por tipo de daño (10). 
sean representativos del conjunto del país, y por otro lado, en el caso de los datos que proceden de las compañías de seguros, al solo contemplar aquellas lesiones obtenidas de denuncias de los usuarios, no sean igualmente representativas del conjunto de lesiones que se dan en los edificios, pues no todas las lesiones llegan a constituir motivo de denuncia en las compañías de seguros.

4) Ninguno de los estudios analizados contiene la concreción suficiente de los procesos patológicos más frecuentes en fachadas y cubiertas para servir como base para la identificación de aquellos factores que puedan favorecer o evitar la aparición de cada una de las lesiones, por lo que se ha planteado recurrir a otras metodologías para complementar la información obtenida.

\section{OBJETIVOS}

Tomando en consideración las conclusiones extraídas, el objetivo de la presente investigación consiste en reconocer las lesiones que más comúnmente presentan los elementos constructivos componentes de la envolvente de los edificios y basándose en los resultados obtenidos, establecer qué factores influyen, de manera relevante, en la durabilidad de los elementos constructivos considerados.

\section{4. ÁMBITO DEL ESTUDIO}

Las fachadas y cubiertas son elementos constructivos expuestos al ambiente exterior, por lo que se requiere de ellos prestaciones más exigentes que en otros elementos, en materia de durabilidad, aislamiento, impermeabilidad y estética.

Si analizamos los procedimientos de diseño de los diferentes elementos constructivos de un edificio, podremos comprobar que en el único elemento en el que la normativa obliga a tener en cuenta la durabilidad en el proceso de diseño, es en los elementos estructurales. Concretamente, la norma EHE o8, a través del Estado Límite de Durabilidad, contempla el fallo de una estructura como consecuencia de los procesos de degradación (11)

Sin embargo, la durabilidad de los elementos constructivos de fachada y cubierta no se tiene en cuenta en el diseño de las mismas más allá de la responsabilidad exigida en la Ley 38/1999 de Ordenación de la Edificación, por la que los agentes deben responder, durante un plazo de tres años, por los daños materiales causados en el edificio por vicios o defectos que afecten a la habitabilidad.

Todo ello es lo que ha motivado que el estudio de la durabilidad se efectúe sobre las fachadas y cubiertas.

\section{METODOLOGÍA DE TRABAJO}

Como ya se ha indicado en las conclusiones reflejadas en el apartado 2, Antecedentes, los resultados obtenidos respecto al conjunto de lesiones más frecuentes en fachadas y cubiertas, no son representativos del conjunto del país, bien por ser locales, o bien por provenir del sector de las compañías de seguros. Además, ninguno de los estudios analizados contiene el detalle requerido sobre los procesos de deterioro, que pueda servir como base para establecer aquellos factores que pueden favorecer o evitar la aparición de cada una de las lesiones. Por lo tanto, se ha considerado necesario recurrir al empleo del método Delphi para completar la información obtenida.

\section{1. ¿En qué consiste el método Delphi?}

El método Delphi, cuyo nombre se inspira en el antiguo oráculo de Delphos, fue ideado a comienzos de los años 50 en el seno del Centro de Investigación estadounidense RAND, como un instrumento para realizar predicciones sobre un caso de catástrofe nuclear. Desde entonces ha sido utilizado frecuentemente como sistema para obtener información sobre incertidumbres de las que no se dispone de datos previos. En la familia de los métodos de pronóstico, habitualmente se clasifica el método Delphi dentro de los métodos cualitativos o subjetivos (12).

Linston y Turoff definen la técnica Delphi como un método de estructuración de un proceso de comunicación grupal que es efectivo a la hora de permitir a un grupo de individuos, como un todo, tratar un problema complejo (13).

El método consiste en una consulta a un grupo de expertos en forma individual por medio de la interacción sucesiva de un cuestionario apoyado por los resultados promedio de la ronda anterior a fin de generar convergencia de opiniones.

Las principales características del método son:

- Utiliza como fuente de información un grupo de personas a las que se supone un conocimiento elevado de la materia que se va a tratar.

- Es un proceso iterativo, como mínimo los expertos deben ser consultados dos veces sobre la misma cuestión, de forma que puedan volver a pensar su respuesta ayudados por la información que reciben de las opiniones del resto de los expertos.

- Mantiene el anonimato de los participantes, o al menos de sus respuestas. Ello permite poder desarrollar un proceso de grupo con unos expertos que no coinciden ni temporal ni espacialmente, de esta forma se evitan las influencias negativas que en las respuestas individuales pudieran tener factores relativos a la personalidad de los expertos participantes.

- Este método se emplea bajo la condición de que no existen datos históricos con los que trabajar.

- La calidad de los resultados depende de la elaboración de los cuestionarios y de la elección de los expertos consultados.

Las ventajas de este método son:

- Alta probabilidad de obtener un consenso en el desarrollo de los cuestionarios sucesivos (lo cual no tiene por qué significar coherencia).

- La información recogida en el curso de la consulta es generalmente rica y abundante.

- Puede utilizarse en cualquier campo de conocimiento.

Los inconvenientes del método son:

- Es largo y costoso. 
Entre las experiencias más relevantes en el uso de este método está la norma ATC-13 Earthquake Damage Evaluation Data for California (14), la cual desarrolla matrices de daño basándose en el método de Delphi.

En materia de predicción de vida útil, una asociación canadiense estableció rangos de durabilidades de elementos constructivos en base al método Delphi (15) mostrándose un ejemplo en la Tabla 6.

\subsection{Aplicación del método Delphi}

Por todo ello se ha considerado que el método Delphi es adecuado para el caso que nos ocupa, por ser un método válido para obtener información sobre incertidumbres de las que no se dispone de datos previos suficientes, habiéndose demostrado efectivo a la hora de permitir a un grupo de expertos en una materia tratar un problema complejo y llegar a una solución consensuada.

La aplicación del método Delphi se ha estructurado mediante dos rondas; es decir, se han efectuado dos instancias de circulación y respuesta de los formularios. Aunque, la formulación teórica del método Delphi propiamente dicho comprende varias etapas sucesivas de envíos de cuestionarios, en buena parte de los casos puede limitarse a dos etapas, lo que sin embargo no afecta a la calidad de los resultados tal como lo demuestra la experiencia acumulada por otros autores en estudios similares (12). A continuación se presentan las etapas seguidas:

1) Selección del grupo de expertos.

2) Elaboración y lanzamiento de los cuestionarios de la primera ronda.

3) Análisis de las respuestas de la primera ronda.

4) Envío de la segunda ronda, informando a cada experto las predicciones de los otros.

5) Recogida y análisis de los resultados de la segunda ronda.

\subsubsection{Selección del grupo de expertos}

En el proceso de selección de los expertos se estableció contacto con los diferentes agentes involucrados en la materia, con el objetivo de que todos ellos estuvieran representados. Se invitó a participar a personas provenientes de universi- dades, institutos de investigación, colegios y asociaciones profesionales, asociaciones empresariales, administraciones públicas, agencias de seguros y profesionales del sector. En total participaron 23 expertos, habiendo representantes de todos los sectores nombrados. Todos los participantes han sido seleccionados por su alto nivel de conocimiento en la materia tratada, contando con expertos de reconocido prestigio y amplia experiencia de cada uno de los campos. Los expertos que han participado no se citan por no vulnerar la ley de protección de datos.

\subsubsection{Elaboración y lanzamiento de los cuestionarios de la primera ronda}

El proceso Delphi comenzó con la convocatoria de los expertos seleccionados para explicarles la finalidad de la información que se les solicitaba y la mecánica iterativa que se seguiría.

Una vez convocados los expertos participantes se procedió al envío de los cuestionarios. El envío fue efectuado mediante correo electrónico. Los cuestionarios fueron cumplimentados y devueltos por los participantes en el plazo estipulado de 15 días. Este proceso se llevó a cabo de forma individual y autónoma, con el objetivo de garantizar el anonimato y evitar que ningún miembro fuera influido por la opinión de otros expertos.

El cuestionario se elaboró de manera que facilitara, en la medida en que una investigación de estas características lo permite, la respuesta por parte de los consultados.

El cuestionario en su primera ronda contenía las siguientes cuestiones:

1) ¿Qué lesiones, en su opinión, se producen con mayor frecuencia en fachadas?

2) ¿Qué lesiones, en su opinión, se producen con mayor frecuencia en cubiertas?

3) De las lesiones que se producen con mayor frecuencia en fachadas, ¿Cuáles de ellas cree usted que preocupan en mayor medida a los usuarios?

4) De las lesiones que se producen con mayor frecuencia en cubiertas, ¿Cuáles de ellas cree usted que preocupan en mayor medida a los usuarios?

Tabla 6. Encuesta sobre vida útil de elementos constructivos en edificios de viviendas utilizada en el desarrollo del método Delphi (15).

\begin{tabular}{|c|c|c|c|c|c|c|c|c|c|c|c|c|c|}
\hline \multirow{2}{*}{$\begin{array}{c}\text { Ref. No. } \\
5.1 \\
\end{array}$} & \multirow{2}{*}{\begin{tabular}{|l|} 
Building Element \\
Roofs (Conventional) \\
\end{tabular}} & \multirow{2}{*}{$\begin{array}{c}\begin{array}{c}\text { Material or } \\
\text { Equipment Type }\end{array} \\
\text { Modified Bitumen }\end{array}$} & \multirow{2}{*}{$\begin{array}{c}\begin{array}{c}\text { Your Estimate } \\
\text { of How Long the } \\
\text { Building Element } \\
\text { will Last }\end{array} \\
\text { to y years }\end{array}$} & \multirow[t]{2}{*}{$\begin{array}{c}\text { Please } \\
\text { Check If No } \\
\text { Opinion }\end{array}$} & \multicolumn{8}{|c|}{$\begin{array}{l}\text { Your Opinion on the } 2 \text { Most } \\
\text { Important Factors Affecting } \\
\text { How Long the Building Part } \\
\text { will Last (Ref. List Below) }\end{array}$} & \multirow[t]{2}{*}{$\begin{array}{c}\text { Please } \\
\text { Check If No } \\
\text { Opinion }\end{array}$} \\
\hline & & & & & $\mathrm{A}$ & $\mathrm{B}$ & $\mathrm{C}$ & $\mathrm{D}$ & $\mathrm{E}$ & $\mathrm{F}$ & G & $\mathrm{H}$ & \\
\hline 5.2 & & Built-up Roofing (BUR) & to__years & & $\mathrm{A}$ & $\mathrm{B}$ & $\mathrm{C}$ & $\mathrm{D}$ & $\mathrm{E}$ & $\mathrm{F}$ & G & $\mathrm{H}$ & \\
\hline $5 \cdot 3$ & & Single-Ply & to years & & $\mathrm{A}$ & $\mathrm{B}$ & $\mathrm{C}$ & $\mathrm{D}$ & $\mathrm{E}$ & $\mathrm{F}$ & G & $\mathrm{H}$ & \\
\hline 5.4 & Roofs (inverted) & Modified Bitumen & to years & & $\mathrm{A}$ & $\mathrm{B}$ & $\mathrm{C}$ & $\mathrm{D}$ & $\mathrm{E}$ & $\mathrm{F}$ & G & $\mathrm{H}$ & \\
\hline 5.5 & & Built-up Roofing (BUR) & to years & & $\mathrm{A}$ & $\mathrm{B}$ & $\mathrm{C}$ & $\mathrm{D}$ & $\mathrm{E}$ & $\mathrm{F}$ & G & $\mathrm{H}$ & \\
\hline 5.6 & & Single-Ply & to _ years & & $\mathrm{A}$ & $\mathrm{B}$ & $\mathrm{C}$ & $\mathrm{D}$ & $\mathrm{E}$ & $\mathrm{F}$ & G & $\mathrm{H}$ & \\
\hline 5.7 & Parapets & Concrete & to $\quad$ years & & $\mathrm{A}$ & $\mathrm{B}$ & $\mathrm{C}$ & $\mathrm{D}$ & $\mathrm{E}$ & $\mathrm{F}$ & G & $\mathrm{H}$ & \\
\hline 5.8 & Base \& Cap Flashing & Sheet Metal & to years & & $\mathrm{A}$ & $\mathrm{B}$ & $\mathrm{C}$ & $\mathrm{D}$ & $\mathrm{E}$ & $\mathrm{F}$ & G & $\mathrm{H}$ & \\
\hline 5.9 & & Non-metalic & to years & & $\mathrm{A}$ & $\mathrm{B}$ & $\mathrm{C}$ & $\mathrm{D}$ & $\mathrm{E}$ & $\mathrm{F}$ & G & $\mathrm{H}$ & \\
\hline 5.10 & Landscaped Terrace & Wood Deck & to years & & $\mathrm{A}$ & $\mathrm{B}$ & $\mathrm{C}$ & $\mathrm{D}$ & $\mathrm{E}$ & $\mathrm{F}$ & G & $\mathrm{H}$ & \\
\hline 5.11 & & Concrete on Raised Floor & to years & & $\mathrm{A}$ & B & $\mathrm{C}$ & $\mathrm{D}$ & $\mathrm{E}$ & $\mathrm{F}$ & G & $\mathrm{H}$ & \\
\hline
\end{tabular}


5) De las lesiones que se producen con mayor frecuencia en fachadas, ¿cuáles de ellas cree usted que pueden afectar a la seguridad?

6) De las lesiones que se producen con mayor frecuencia en cubiertas, ¿̇cuáles de ellas cree usted que pueden afectar a la seguridad?

7) Por su experiencia, de las lesiones que se producen con mayor frecuencia en fachadas, ¿̇cuáles de ellas requieren de una mayor inversión para su reparación?

8) Por su experiencia, de las lesiones que se producen con mayor frecuencia en cubiertas, ¿̇cuáles de ellas requieren de una mayor inversión para su reparación?

9) ¿Qué factores cree usted que deben considerarse al evaluar la vida útil de una fachada?

10) ¿Qué factores cree usted que deben considerarse al evaluar la vida útil de una cubierta?

11) ¿Qué factores cree usted que pueden incidir en el deterioro temprano de una fachada?

12) ¿Qué factores cree usted que pueden incidir en el deterioro temprano de una cubierta?

Los expertos disponían de diez casillas asignadas a cada pregunta donde podían contestar un máximo de diez opciones, debiendo de ordenar las respuestas por orden de importancia.

\subsubsection{Análisis de las respuestas de la primera ronda}

Tras la recepción de los resultados de la primera ronda se procedió a su análisis. En cada pregunta se seleccionaron las 10 o 5 respuestas más repetidas, dependiendo de la pregunta, y se ordenaron según el número de veces que habían sido seleccionadas por un experto. Las respuestas que coincidían en número se ordenaron en función de la puntuación que cada uno de los expertos asignó a cada respuesta.

\subsubsection{Envío de la segunda ronda, informando a cada experto de las predicciones de los otros}

Después de procesar los resultados obtenidos del primer cuestionario, se comenzó la segunda ronda. En el cuestionario correspondiente se incluyó en cada pregunta las respuestas que más fueron elegidas en la primera ronda. En cada pregunta, el experto podía tachar o eliminar aquellas respuestas con las que no estaba de acuerdo, añadir las que creía que se habían omitido y deberían constar, y cambiar el orden de las que se incluían, quedando las respuestas ordenadas de mayor a menor relevancia, en función de lo que se estaba preguntando.

El método Delphi intenta llegar a pronósticos lo más consensuados posibles a través del intercambio de razones y argumentos; sin embargo, ello no debe conducir a que los expertos se sientan obligados o presionados en absoluto a plegarse a las respuestas mayoritarias. Sólo si las respuestas mayoritarias y los argumentos esgrimidos durante las discusiones y presentaciones convencen al experto, éste deberá modificar su respuesta.
5.2.5. Recogida y análisis de resultados de la segunda ronda

Los cuestionarios de la segunda ronda fueron cumplimentados y devueltos por los participantes en el plazo estipulado de 10 días. De la misma forma que en la etapa anterior, se procedió a seleccionar las diez respuestas más repetidas entre los participantes ordenándose según importancia otorgada.

A continuación se muestran los resultados obtenidos:

1. ¿Qué lesiones, en su opinión, se producen con mayor frecuencia en fachadas?

- Desprendimientos de revestimientos discontinuos o elementos de adorno

- Grietas y fisuras

- Fisuras y/o desprendimientos en revestimientos continuos

- Humedades de filtración a través del paño ciego o a través de sus juntas

- Humedades de condensación intersticiales y/o superficiales

- Humedad por falta de estanqueidad en carpinterías

- Oxidación y/o corrosión de elementos metálicos

- Eflorescencias

- Deterioro del aspecto exterior: suciedad, variación de color, manchas de óxido, etc.

- Degradación de los materiales tales como erosiones o costras

2. ¿Qué lesiones, en su opinión, se producen con mayor frecuencia en cubiertas?

- Humedades por filtración de agua

- Humedades por condensación

- Obstrucción de sumideros y canalones por falta de mantenimiento

- Rotura, deterioro y/o desplazamiento de la capa de impermeabilización

- Falta de juntas de dilatación o mala ejecución y/o mantenimiento de las existentes

- Degradación del material de protección en cubiertas planas

- Desprendimiento de la protección en cubiertas inclinadas

- Fisuras en antepechos

- Filtración de agua a través de puntos singulares (encuentros con paramentos verticales, etc...)

- Grietas y fisuras

3. De las lesiones que se producen con mayor frecuencia en fachadas, ¿Cuáles de ellas cree usted que preocupan en mayor medida a los usuarios?

- Desprendimiento de acabados y elementos sueltos

- Humedades

- Fisuras, grietas, desplomes o deformaciones excesivas

- Eflorescencias, presencia de moho, decoloración, suciedad...etc

- Corrosión de armaduras

4. De las lesiones que se producen con mayor frecuencia en cubiertas, ¿Cuáles de ellas cree usted que preocupan en mayor medida a los usuarios?

- Humedades por filtración de agua

- Desprendimiento de la protección cubiertas inclinadas o albardillas en cubiertas planas 
- Humedades de condensación

- Deformaciones del elemento estructural soporte

- Grietas y/o fisuras

5. De las lesiones que se producen con mayor frecuencia en fachadas, ¿cuáles de ellas cree usted que pueden afectar a la seguridad?

- Desprendimientos de revestimientos o elementos sueltos

- Fisuras, grietas, desplomes o deformaciones excesivas

- Humedades

- Procesos de corrosión

- Falta de estabilidad geométrica del cerramiento sobre el forjado

6. De las lesiones que se producen con mayor frecuencia en cubiertas, ¿̇cuáles de ellas cree usted que pueden afectar a la seguridad?

- Humedades por filtración de agua

- Desprendimiento de la protección en cubiertas inclinadas o albardillas en cubiertas planas

- Inestabilidad de los antepechos por dilatación y contracción del tablero de cubierta

- Rotura y/o desprendimiento de elementos anclados

- Colapso por exceso de peso

7. Por su experiencia, de las lesiones que se producen con mayor frecuencia en fachadas, ¿cuáles de ellas requieren de una mayor inversión para su reparación?

- Reparación o sustitución del acabado discontinuo

- Grietas y/o deformaciones por movimientos estructurales o asentamiento de cimientos

- Reparación o sustitución del acabado continuo

- Humedades de filtración generalizadas

- Humedades de condensación por fallo en el aislamiento térmico

8. Por su experiencia, de las lesiones que se producen con mayor frecuencia en cubiertas, ¿̇cuáles de ellas requieren de una mayor inversión para su reparación?

- Humedades por filtración de agua

- Reparación o sustitución de la protección en cubiertas inclinadas

- Lesiones ocasionadas por sobrecargas

- Reparación o sustitución de la protección en cubiertas planas

- Mejora del aislamiento térmico

9. ¿Qué factores cree usted que deben considerarse al evaluar la vida útil de una fachada?

- Tipología constructiva

- Calidad y durabilidad de los materiales empleados, especialmente de los de revestimiento

- Impermeabilidad del sistema y correcto diseño del sistema de evacuación del agua

- Nivel de calidad de la ejecución y de su control

- El diseño de juntas de movimiento, dilatación y retracción

- Aislamiento térmico evitando puentes térmicos

- Agentes climatológicos (lluvia, viento, soleamiento...)

- Realización y seguimiento de un plan de mantenimiento

- Localización del inmueble (zona geográfica, situación de exposición, orientación...)

- Contaminación ambiental
10. ¿Qué factores cree usted que deben considerarse al evaluar la vida útil de una cubierta?

- Tipología constructiva

- Calidad y durabilidad de los materiales empleados, especialmente de los de protección

- Impermeabilidad del sistema

- Correcto diseño del sistema de evacuación de agua: inclinación de faldones, sección de canalones ...

- Nivel de calidad de la ejecución y de su control

- El diseño de juntas de movimiento, dilatación y retracción

- Uso

- Agentes climatológicos (lluvia, viento, soleamiento...)

- Localización del inmueble (zona geográfica, situación de exposición, orientación...)

- Realización y seguimiento de un plan de mantenimiento

11. ¿Qué factores cree usted que pueden incidir en el deterioro temprano de una fachada?

- Durabilidad de los materiales constituyentes del cerramiento, especialmente los de revestimiento

- Defectos de ejecución

- Falta de mantenimiento

- Agentes climatológicos

- Error de proyecto

- Mal diseño de juntas de movimiento, dilatación y retracción

- Tipología constructiva

- Mal diseño del sistema de evacuación de agua

- Falta del anclaje o de adherencia adecuados de los acabados

- Contaminación medioambiental

12. ¿Qué factores cree usted que pueden incidir en el deterioro temprano de una cubierta?

- Falta de mantenimiento

- Defectos de ejecución

- Durabilidad de los materiales constituyentes de la cubierta, especialmente los de protección

- Error de proyecto

- Agentes climatológicos

- Mal diseño del sistema de evacuación de aguas

- Mal diseño de juntas de movimiento, dilatación y retracción

- Uso inadecuado

- Tipología constructiva

\section{ANÁLISIS DE LOS RESULTADOS}

\subsection{Lesiones que se producen con más frecuencia en la envolvente del edificio}

Tras la aplicación del método Delphi, los resultados obtenidos, no contradicen a los conseguidos mediante el análisis bibliográfico desarrollado, pero sí son más concretos y abarcan el ámbito nacional. De esta forma se ha salvado la barrera encontrada inicialmente de contar únicamente con estudios sesgados, bien por ser locales, o bien por provenir del sector de las compañías de seguros.

Los resultados alcanzados son el punto de partida para poder establecer estrategias nacionales de prevención en relación a la presencia de lesiones, que deberán abarcar las diferentes fases del proceso de construcción: 
- Diseño: En esta fase la estrategia de prevención debe ir enfocada a incluir, en los planes de estudio de aquellos títulos universitarios vinculados a la construcción de edificios, el estudio de la patología y la prevención de lesiones, profundizando en la necesidad del correcto diseño de los elementos constructivos. Por otro lado, de cara a paliar la posible falta de formación de los profesionales en activo en este ámbito, es necesario fomentar cursos formativos desde los organismos competentes en la materia.

- Ejecución: El control de la ejecución en obra deberá contemplar aquellas inspecciones necesarias para evitar los errores que pueden inducir a la aparición de las lesiones más frecuentes.

- Uso: Durante la fase de uso del edificio los procedimientos de inspección normativos deben contemplar la identificación y caracterización de daños, para lo que se hace necesario profundizar en la formación de los técnicos responsables para el reconocimiento de los síntomas, posibles causas y las opciones para la reparación.

\subsection{Percepción de los usuarios de las diferentes lesiones}

La importancia que los usuarios que habitan los edificios le dan a una lesión va en relación a dos parámetros: apariencia y seguridad. Esta afirmación se deduce a partir de las lesiones que preocupan a los usuarios en mayor medida: humedades, eflorescencias, moho, decoloración, suciedad...etc., por un lado, y desprendimientos, fisuras, grietas y deformaciones por otro. La percepción que tienen los usuarios de las lesiones tiene gran influencia en la ciencia de la patología ya que la importancia que un usuario de a una lesión será el motivo que le lleve a poner los medios necesarios para su reparación. Las acciones que se concluye son necesarias a partir de los resultados obtenidos son, por un lado, la promoción de inspecciones que permitan detectar lesiones que los usuarios no perciben como relevantes y que, sin embargo, pueden actuar en detrimento de la seguridad, y por otro lado, el desarrollo de campañas informativas que faciliten a los usuarios o a aquellas empresas y/u operarios a cargo del mantenimiento de los edificios, el detectar los síntomas de las lesiones más frecuentes.

\subsection{Análisis de la inversión necesaria para la reparación de las lesiones}

Las lesiones que han sido señaladas por los expertos como aquellas que requieren mayor inversión para su reparación se pueden clasificar en tres grupos. El primero incluiría las lesiones que destinan un elevado porcentaje del presupuesto a medios auxiliares como son los andamios, por ejemplo, la reparación de daños generalizados en el revestimiento de la fachada o en la protección de la cubierta. El segundo engloba a aquellos daños que implican la reparación de elementos estructurales. Por último, aquellas lesiones relacionadas con la humedad de filtración y condensación, elección esta última motivada por ser lesiones dónde la propia diagnosis puede suponer un coste elevado en sí misma y que abarcan, generalmente, diferentes capas del sistema constructivo, lo que eleva el coste de la intervención.

Con el objetivo de reducir el coste del ciclo de vida del edificio en su conjunto y de los sistemas constructivos de fachada y cubierta en particular, los planes de mantenimiento incluidos en el libro del edificio deben prestar especial atención a la prevención de las lesiones más comunes, destacando aquellas que necesiten una elevada inversión para su reparación o aquellas que a su vez sean la causa de nuevas lesiones diferentes a la original o agraven las ya existentes. Sólo mediante la adecuada regulación de la durabilidad y el mantenimiento de las soluciones constructivas se podrá corregir la tendencia creciente en cuanto a costes de mantenimiento del patrimonio edificado (16).

\subsection{Factores que afectan a la durabilidad de la envolvente}

Desde principios del siglo XX, se han editado una serie de normas ISO en relación a la planificación de la vida útil de los edificios, en concreto, las normas ISO 15686. En su parte ocho, ISO 15686-8 Reference service life (17), proporciona criterios para el cálculo de vida útil estimada utilizando el método de los factores. Éste utiliza un enfoque determinista permitiendo cuantificar la vida útil de un elemento o sistema, sujeto a unas condiciones específicas, partiendo de la vida útil de referencia y modificándola mediante unos factores relativos a las condiciones específicas de cada caso. El método contempla los siguientes factores:

- Factor A: Calidad de los componentes

- Factor B: nivel de diseño

- Factor C: nivel de calidad en la ejecución de las obras

- Factor D: condiciones interiores

- Factor E: condiciones de exposición exteriores

- Factor F: condiciones de uso

- Factor G: nivel de mantenimiento

Los factores que los expertos participantes en el estudio han señalado como aquellos que en mayor medida afectan a la durabilidad de la envolvente del edificio, coinciden con los especificados en la norma ISO 15686-8. A continuación se clasifican los factores aportados por el panel de expertos en función de los apartados que indica la norma:

\section{Factor A: Calidad de los componentes}

- Calidad y durabilidad de los materiales empleados

\section{Factor B: nivel de diseño}

- Tipología constructiva

- Impermeabilidad del sistema y correcto diseño del sistema de evacuación del agua

- El diseño de juntas de movimiento, dilatación y retracción

- Aislamiento térmico evitando puentes térmicos

Factor C: nivel de calidad en la ejecución de las obras - Nivel de calidad de la ejecución y de su control

\section{Factor D: condiciones interiores}

Factor E: condiciones de exposición exteriores

- Agentes climatológicos (lluvia, viento, soleamiento...)

- Localización del inmueble (zona geográfica, situación de exposición, orientación...)

- Contaminación ambiental

\section{Factor F: condiciones de uso}

- Uso

\section{Factor G: nivel de mantenimiento}

- Realización y seguimiento de un plan de mantenimiento 
El único factor que los expertos consultados no marcan como especialmente relevante, en el caso concreto de la envolvente del edificio, es el que hace referencia a las condiciones interiores del mismo.

En consecuencia, se puede concluir que las áreas establecidas por la norma como aquellas que deben cubrir el conjunto de factores son un buen punto de partida en relación a establecer factores específicos que afectan a la durabilidad de cada sistema constructivo. La publicación de la norma ISO 15686 ha supuesto un gran avance en el establecimiento de unas líneas generales que permiten identificar los factores que afectan a la durabilidad de los elementos constructivos. No obstante, aún quedan cuestiones muy importantes como es la caracterización de los mecanismos y factores de deterioro de los sistemas constructivos propios de cada país.

\section{CONCLUSIONES}

Es obvio el interés que despierta en los diferentes países conocer datos de las lesiones que presentan sus edificios, determinar las posibles causas y extraer las oportunas conclusiones de cara a mejorar la calidad de la construcción. Así lo demuestran las múltiples estadísticas sobre patología elaboradas en nuestro país y expuestas en el apartado 2, dónde se concluye que las lesiones que más afectan a las fachadas son las humedades, las fisuras, grietas y desprendimientos; en el caso de las cubiertas son las humedades, las fisuras y las grietas los daños señalados en general como más frecuentes.

Estos estudios son un buen inicio para poner de manifiesto dónde pueden localizarse los fallos, sin embargo, dado que son, o bien estudios locales, o bien se basan en datos procedentes de compañías de seguros, sus conclusiones no son representativas del conjunto de lesiones que se dan en los edificios a nivel nacional, y por lo tanto se hace necesario el llevar a cabo estudios que permitan obtener una perspectiva de la patología a nivel estatal.

En este contexto, se ha empleado el método Delphi con el objetivo de aportar una perspectiva de la patología a nivel nacional, inexistente hasta el momento, enumerándose aquellas lesiones que los expertos señalan como más frecuentes. Asimismo se han abordado cuestiones tales como la percepción de los usuarios de las diferentes daños y cuáles de ellos conllevan mayores costes de reparación.

El método Delphi demuestra ser una herramienta útil para obtener información sobre incertidumbres de las que no se dispone de datos previos, resultando ser muy efectivo a la hora de permitir a un grupo de individuos, como un todo, tratar un problema complejo.

Tras analizar los resultados de las diferentes cuestiones abordadas en la aplicación del método se concluye que existe una latente necesidad de:

- Reforzar la formación que reciben los profesionales del sector de la construcción en materia de patología (lesiones frecuentes, sus síntomas, prevención, reparación...)

- Adecuar los procedimientos de control en obra a la detección de daños más comunes.

- Establecer procedimientos de inspección normativos que contemplen la identificación y caracterización de daños, para lo que se hace necesario profundizar en la formación de los técnicos responsables para el reconocimiento de los síntomas, posibles causas y las opciones para la reparación.

- Generar reglamentación y guías de apoyo en relación al mantenimiento y la durabilidad de edificios de cara a prevenir la aparición de lesiones.

Se ha constatado como la norma ISO 15686 establece unas líneas generales para identificar los factores que afectan a la durabilidad de los elementos constructivos, pero se detecta la necesidad de efectuar estudios más concretos que permitan identificar los factores relevantes que condicionan la vulnerabilidad de los elementos constructivos propios de cada país.

Los resultados obtenidos del estudio llevado a cabo permiten dar un paso en el campo de la durabilidad de los sistemas constructivos a través del análisis de su vulnerabilidad. El conocer los mecanismos de deterioro que se dan con más frecuencia permite establecer pautas de diseño que mejoren la respuesta de los sistemas actuales al medio, aumentando así su vida útil. Las repercusiones en el ámbito económico que estudios de este tipo pueden reportar, pueden ser importantes, al incidir en la mejora de calidad de materiales del proceso constructivo y de la propia calidad de los proyectos, sin despreciar el ahorro que se obtendría evitando costosas reparaciones, que en algunos casos han de constituir un refuerzo generalizado del edificio, o en otros una demolición prematura.

\section{REFERENCIAS}

(1) Moser, K., Hovde, P. J. (2004). Performance based methods for service life prediction. Rotterdam: CIB.

(2) Monjo-Carrió, J. (2007). Durabilidad vs Vulnerabilidad. Informes de la Construcción, 59(507): 43-58, doi: http:// dx.doi.org/10.3989/ic.2007.v59.i507.531.

(3) Departamento de construcción de la ETSAV. (1987). Patología de fachadas urbanas. Valladolid: Universidad de Valladolid.

(4) Monjo-Carrió, J. (1992). Chequeo constructivo de fachadas de Madrid/España. Informes de la construcción, 43(418): 35-52, doi: http://dx.doi.org/10.3989/ic.1992.v43.i418.1353.

(5) Escribano-Villán, J. (1994). Siniestralidad arquitectónica. Bilbao: Colegio Oficial de Arquitectos Vasco-Navarro.

(6) Aragón-Fitera, J. (2010). Análisis estadístico de la patología de forjados de hormigón armado. La Coruña: Universidad de la Coruña.

(7) Pérez-Navarro, J. (200o). La casa en forma. Desarrollo y conclusiones de la campaña de inspecciones técnicas de edificios. Murcia: Colegio oficial de Aparejadores y Arquitectos Técnicos de Murcia. 
(8) Úbeda-de Mingo, P. (2001). Rehabilitación y mantenimiento de edificios. Madrid: Escuela universitaria de arquitectura técnica de Madrid.

(9) Región de Murcia. (2010). Anexo técnico de la IV edición de los premios de Calidad en la Edificación de la Región de Murcia. Murcia: Consejería de Obras Públicas y Ordenación del Territorio.

(10) Rosa-Roca, N. (2012). Manual de prevención de fallo: Entanqueidad en fachadas. Murcia: Colegio Oficial de Aparejadores, Arquitectos Técnicos e Ingenieros de Edificación de la Región de Murcia.

(11) Ministerio de Fomento. (2008). Real Decreto 1247/2008, de 18 de julio, por el que se aprueba la Instrucción hormigón estructural (EHE-08). Boletín Oficial del Estado, n 203. España.

(12) Astigarraga, E. (2011). El método Delphi. San Sebastian: Facultad de CC.EE. y Empresariales-Universidad de Deusto.

(13) Linstone, H., Turoff, M. (1975). The Delphi Method. Techniques and Applications. Addison-Wesley.

(14) Rojahn, C., Sharpe, R. (1985). ATC 13: Earthquake Damage Evaluation Data for California. Redwood City: Applied technology council.

(15) CMHC. (2000). Service Life of Multi-Unit Residential Building, Elements and Equipment. Ontario: Canada Mortgage and Housing Corporation.

(16) Avellaneda i Díaz-Grande, J. (1983). Medio ambiente, comportamiento y durabilidad del cerramiento. Informes de la contrucción, 35(355-356): 109-113.

(17) ISO. (2008). ISO 15686-8 Buildings and constructed assets - Service life planning: Part 8, Reference service life. Ginebra: International Organization for Standardization. 